\title{
VIII. - ESTIMATION DE LA VALEUR ALIMENTAIRE ET DIGESTIBILITÉ DU MÄ̈̈S GRAIN CHEZ LE PORCELET ET LE RAT
}

\author{
A. AUMAITRE et P. PAIN \\ avec la collaboration de M. MARion et Jany Peiniau \\ Station de Recherches sur l'Élevage des Porcs, \\ Centre national de Recherches zootechniques, I. N. R. A., \\ 78 - Jouy-en-Josas

\section{RÉSUMÉ}

L'étude de la valeur nutritionnelle du maïs grain séché à deux températures (80 et $14^{\circ}$ ) associée à 3 traitements de conservation a été entreprise sur le porcelet et le rat récemment sevrés.

L'influence du traitement a été mesurée par les performances zootechniques des animaux (croissance, consommation d'aliment, indice de consommation, état sanitaire); ainsi que sur la digestibilité des principaux éléments de la ration.

L'effet de la température paraft faible ou nul sur les performances zootechniques et la digestibilité des éléments de la ration chez le porcelet. Par contre, chez le rat qui recevait une ration très riche en mais $\left(93.5 \mathrm{p}\right.$. Ioo) on note un effet dépressif de la température $\left(\mathrm{I}_{4} \mathrm{O}^{\circ}\right)$ sur le coefficient d'utilisation digestive des protéines de 4 p. Ioo.

L'effet le plus significatif paraît être l'échauffement du maîs qui provoque une diminution de la vitesse de croissance de 5 à $40 \mathrm{p}$. roo suivant les régimes et les animaux. Les performances sont expliquées à la fois par une diminution de la quantité d'aliment consommée (4 à 20 p. roo) et par une augmentation de l'indice de consommation qui peut atteindre 13 p. Ioo par rapport à un maiss témoin séché dès la récolte.

Des conclusions sont tirées relativement au choix des méthodes de séchage, et aux modalités expérimentales de mise en évidence rapide de l'altération de la valeur alimentaire du maîs : le rat semble être un animal de choix pour l'appréciation rapide, en 3 jours, de la dépréciation de la valeur alimentaire du mais traité.

\section{I. - INTRODUCTION}

La mise en cuvre de la chaleur, outre la déshydratation qu'elle provoque, peut entraîner une altération du grain et une modification de sa valeur alimentaire pour le monogastrique (HATHAWAY et al., I952). L'effet de tels traitements sur 1'utilisation du grain par les animaux est encore mal connu : seules quelques mesures ont 
été faites chez le Porc par CABELI et al. (I958) et JENSEN et al. (I960), utilisant une température au maximum de $100^{\circ} \mathrm{C}$. De plus, on ignore si le séchage à des hautes températures a une influence sur la digestibilité des éléments de la ration.

Nous avons ainsi réalisé une expérience sur porcelets sevrés précocement recevant une ration à base de maîs séché artificiellement et comparé la croissance des animaux ainsi que la digestibilité des principaux éléments de la ration.

Nous avons de plus réalisé une expérience sur le jeune rat au sevrage en vue d'utiliser un animal de laboratoire pour estimer les effets de quelques modes de séchage du maïs sur sa valeur nutritionnelle.

\section{II. - MATÉRIEL E'T MÉTHODES}

\section{A. - Expériences sur porcelets}

Vingt portées de porcelets de race Large White, sevrés à 5 semaines sont répartis en lots de 2 porcelets ( 1 mâle, I femelle, pleins frère-sœur) à raison de 3 traitements par portée suivant le schéma des blocs incomplets"équilibrés de type I répété une seule fois (CocHRAN et Cox, I962). Chaque lot tiré au sort reçoit l'un des 6 régimes présentés au tableau $I$; le maïs ayant subi différents traitements constitue la seule céréale de la ration. On a mesuré les performances zootechniques des animaux (croissance, consommation d'aliment). De plus, 30 porcelets provenant de 5 portées, comportant des animaux de poids très homogènes ont été placés en cages de digestibilité. On a mesuré sur ces animaux au cours de 3 périodes successives de I 5 jours le CUD de la matière sèche et des protéines des aliments expérimentaux ainsi que le coefficient de rétention de l'azote.

\section{TABLEAU I}

Composition des régimes expérimentaux pour porcelets (p. 100) et contrôle des aliments

\begin{tabular}{|c|c|c|c|c|c|c|}
\hline Régime ou lot no & 1 & 2 & 3 & 4 & 5 & 6 \\
\hline 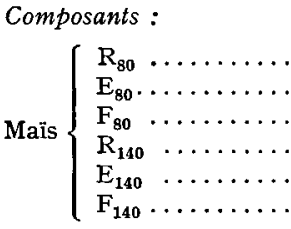 & 65,5 & 65,5 & 65,5 & 65,5 & 65,5 & 65,5 \\
\hline $\begin{array}{l}\text { Tourteau de soja ...... } \\
\text { Farine de poisson ..... } \\
\text { Poudre de lait écrémé } \\
\text { Huile d'arachide. ...... } \\
\text { Minéraux et vitamines . }\end{array}$ & $\begin{array}{l}21 \\
3 \\
5 \\
1,5 \\
4\end{array}$ & $\begin{array}{l}21 \\
3 \\
5 \\
1,5 \\
4\end{array}$ & $\begin{array}{l}21 \\
3 \\
5 \\
1,5 \\
4\end{array}$ & $\begin{array}{l}21 \\
3 \\
5 \\
1,5 \\
4\end{array}$ & $\begin{array}{l}21 \\
3 \\
5 \\
1,5 \\
4\end{array}$ & $\begin{array}{l}21 \\
3 \\
5 \\
1,5 \\
4\end{array}$ \\
\hline $\begin{array}{c}\text { Dosage des aliments: } \\
\text { Matière sèche, \% } \\
\text { Protéines brutes, \% }\end{array}$ & $\begin{array}{l}89,12 \\
20,2\end{array}$ & $\begin{array}{l}89,33 \\
20,2\end{array}$ & $\begin{array}{l}89,66 \\
20,4\end{array}$ & $\begin{array}{l}89,66 \\
20,2\end{array}$ & $\begin{array}{l}91,14 \\
20,4\end{array}$ & $\begin{array}{l}90,36 \\
20,5\end{array}$ \\
\hline
\end{tabular}




\section{B. - Expérience sur rats}

Cinquante-quatre rats blancs âgés de 4 semaines sont répartis en 6 lots aussi comparables que possible (HENRY, I968), placés en cage individuelle et soumis à des régimes expérimentaux très riches en maïs, mais rééquilibrés en acides aminés essentiels, pour permettre une croissance normale (tabl. 2 et 2 bis). Sur ces animaux, on a mesuré les performances pondérales, la consommation d'aliment ainsi que la digestibilité des aliments (sur 4 rats de même poids par lot).

Enfin, en vue de déterminer l'utilisation métabolique de l'azote, le broyage global des carcasses des 6 lots de rats a été réalisé et on a calculé le coefficient d'utilisation pratique de l'azote des rations.

\section{TABLEAU 2}

Composition des aliments pour la mesure de la valeur nutritive pour le rat (p. roo)

\begin{tabular}{|c|c|c|c|c|c|c|}
\hline Régime & 1 & 2 & 3 & 4 & 5 & 6 \\
\hline 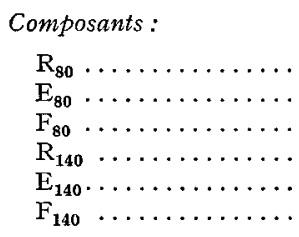 & 93,5 & 93,5 & 93,5 & 93,5 & 93,5 & 93,5 \\
\hline $\begin{array}{l}\text { Vitamines } \ldots \ldots \ldots \ldots \\
\text { Minéraux } \ldots \ldots \ldots \ldots \\
\text { Acides aminés synthé- } \\
\text { tiques } \ldots \ldots \ldots \ldots \ldots\end{array}$ & $\begin{array}{l}1 \\
4,5 \\
1,16\end{array}$ & $\begin{array}{l}1 \\
4,5 \\
1,16\end{array}$ & $\begin{array}{l}1 \\
4,5 \\
1,16\end{array}$ & $\begin{array}{l}1 \\
4,5 \\
1,16\end{array}$ & $\begin{array}{l}1 \\
4,5 \\
1,16\end{array}$ & $\begin{array}{l}1 \\
4,5 \\
1,16\end{array}$ \\
\hline $\begin{array}{l}\text { Dosage des aliments : } \\
\text { Matière sèche } \ldots \ldots \\
\text { Protéines } \ldots \ldots \ldots\end{array}$ & $\begin{array}{r}88,21 \\
8,56\end{array}$ & $\begin{array}{r}88,55 \\
9,44\end{array}$ & $\begin{array}{r}88,31 \\
9,18\end{array}$ & $\begin{array}{r}89,13 \\
9,68\end{array}$ & $\begin{array}{r}89,84 \\
9,31\end{array}$ & $\begin{array}{r}89,28 \\
8,86\end{array}$ \\
\hline
\end{tabular}

\section{TABLEAU 2 bis}

Supplémentation des régimes du rat en acides aminés de synthèse (p. Ioo de la ration (1))

\begin{tabular}{|c|c|c|c|c|}
\hline Acides aminés & $\begin{array}{c}\text { Besoins de } \\
\text { croissance } \\
\text { du rat } \\
\text { (N. R. C., } \\
\text { 1962) }\end{array}$ & $\begin{array}{c}\text { Apport } \\
\text { du mais } \\
\text { (Pron, } \\
\text { 1964) }\end{array}$ & $\begin{array}{c}\text { Supplé- } \\
\text { mentation } \\
(+)\end{array}$ & $\begin{array}{l}\text { Apport } \\
\text { théorique } \\
\text { total }\end{array}$ \\
\hline L-lysine & 0,90 & 0,18 & 0,60 & 0,78 \\
\hline L-thréonine .. & 0,50 & 0,36 & 0,10 & 0,46 \\
\hline L-tryptophane $\ldots \ldots \ldots \ldots$ & 0,15 & 0,09 & 0,06 & 0,15 \\
\hline DL-méthionine + cystine ... & 0,50 & 0,18 & 0,30 & 0,48 \\
\hline L-Isoleucine $\ldots \ldots \ldots \ldots \ldots$ & 0,55 & 0,40 & 0,10 & 0,50 \\
\hline
\end{tabular}

(1) Supplémentation à une dose sub-optimum par rapport aux besoins pour assurer une croissance normale. 


\section{C. - Calculs et interprétation statistique}

L'analyse des résultats zootechniques obtenus sur porcelets a été réalisée par interprétation du schéma en blocs incomplets équilibrés du type I, appliqué à un dispositif factoriel $3 \times 2$ par M. J. M. ReGNier ('). L'analyse des autres résultats (digestibilité) a été effectuée par analyse de variance simple et par comparaison multiple des moyennes selon la méthode de Duncan.

\section{III. - RÉSUL'TATS}

\section{I. - Expérience sur porcelets}

a) Performances zootechniques des animaux.

On constate un effet dépressif sur le gain de poids moyen avec les régimes à base de maïs échauffé ; par contre, la technique de séchage ne semble pas jouer (tabl. 3).

\section{TABLEAU 3}

Performances zootechniques et état sanitaire des porcelets

(Io répétitions de 2 porcelets par lots)

\begin{tabular}{|c|c|c|c|c|c|c|}
\hline \multirow{2}{*}{ Régime } & \multicolumn{3}{|c|}{$80^{\circ}$} & \multicolumn{3}{|c|}{$140^{\circ}$} \\
\hline & $\mathrm{R}$ & $\mathrm{E}$ & $\mathrm{F}$ & $\mathbf{R}$ & $\mathbf{E}$ & $\mathbf{F}$ \\
\hline Gain moyen $\mathrm{g} / \mathrm{j}$ & $372^{b}$ & $348^{a}$ & $370^{b}$ & $385^{b}$ & $330^{a}$ & $380^{b}$ \\
\hline Consommation $\mathrm{g} / \mathrm{j}$ & $596^{a b c}$ & $590^{a b}$ & $577^{a}$ & $638^{b c}$ & $574^{a}$ & $639 c$ \\
\hline Indice de consommation. $\mathrm{kg} / \mathrm{kg}$ & $1,68^{b c}$ & $1,68^{b c}$ & $1,55^{c}$ & $1,67 b c$ & $1,76^{a}$ & $1,70^{b c}$ \\
\hline Jours de diarrhée $\left({ }^{(}\right) \ldots \ldots \ldots$ & $3,5^{c}$ & $6,2^{a}$ & $4,0^{c}$ & $5,8^{a b}$ & $4,3^{b c}$ & $4,1^{a}$ \\
\hline
\end{tabular}

(1) Nombre moyen de jours de diarrhée/porcelet/mois de période expérimentale.

$a, b, c \ldots$ les moyennes affectées des mêmes lettres ne sont pas significativement différentes entre elles.

I1 n'existe d'autre part pas d'interaction significative entre la température et le mode de traitement; les performances de croissance ne sont pas sensiblement différentes entre elles pour 4 des traitements. De même, les deux plus mauvais traitements ne diffèrent pas entre eux.

Les quantités moyennes d'aliments consommées par jour varient dans des limites plus faibles, mais sensiblement dans le même sens que les performances pondérales, avec un effet dépressif plus net de l'échauffement lorsqu'il est associé à une température de séchage élevée.

L'indice de consommation calculé pour les différents régimes est légèrement plus élevé pour les lots séchés à $I 40^{\circ}$ bien que 1 " " effet température " ne soit pas significatif. Cependant, il constitue un aliment médiocre car le maïs échauffé provoque

(1) Ingénieur au Cneema 92 - Antony. 
lorsqu'il est traité à température élevée un indice de consommation significativement supérieur à tous les autres.

L'appréciation de l'état sanitaire des animaux montre que tous les régimes semblent favoriser la diarrhée. Cependant, ni les conditions de stockage, ni les températures de séchage ne semblent jouer un rôle sur la fréquence de la diarrhée. Le maïs échauffé $\left(E_{80}\right)$ favorise encore une fois un état sanitaire médiocre.

b) Utilisation digestive des principaux éléments de la ration.

Les animaux placés individuellement en cage et alimentés en quantité égalisée présentent des performances de croissance comparables suivant les lots aux résultats précédents (tabl. 4).

\section{TABLEAU 4}

Coefficients d'utilisation digestive apparente et performances des porcelets en cage de digestibitité ( 6 porcelets par lot)

\begin{tabular}{|c|c|c|c|c|c|c|}
\hline \multirow{2}{*}{ Régime } & \multicolumn{3}{|c|}{$80^{\circ}$} & \multicolumn{3}{|c|}{$140^{\circ}$} \\
\hline & $\mathrm{R}$ & $\mathrm{E}$ & $\mathrm{F}$ & $\mathbf{R}$ & $\mathrm{E}$ & $\mathrm{F}$ \\
\hline $\begin{array}{l}\text { Gain de poids } g / j \ldots \ldots \ldots \ldots \\
\text { Consommation } g / j \text { ( }\left(^{(1)} \ldots \ldots \ldots \ldots\right. \\
\text { Indice de consommation } \ldots \ldots \ldots \\
\text { Efficacité protidique }\left({ }^{2}\right) \ldots \ldots \ldots\end{array}$ & $\begin{array}{l}434^{b} \\
584 \\
1,35^{c} \\
3,70^{c}\end{array}$ & $\begin{array}{l}392^{a} \\
568 \\
1,45^{a b} \\
3,42^{a b}\end{array}$ & $\begin{array}{l}409 a b \\
586 \\
\quad 1,38^{a b c} \\
3,60^{b c}\end{array}$ & $\begin{array}{l}396^{a} \\
585 \\
1,47^{a} \\
3,39^{a b}\end{array}$ & $\begin{array}{l}378^{1} \\
554 \\
1,47^{a} \\
3,35^{a}\end{array}$ & $\begin{array}{l}424^{b} \\
581 \\
1,37^{b c} \\
3,63^{b c}\end{array}$ \\
\hline $\begin{array}{l}\text { Coefficient de digestibilité, p. } 100 \\
\text { Matière sèche } \ldots \ldots \ldots \ldots \ldots \ldots \ldots \\
\text { Protéines........... } \\
\text { Rétention azotée, p. } 100 \ldots \ldots \ldots\end{array}$ & $\begin{array}{l}86,5 \\
83,9 \\
52,8\end{array}$ & $\begin{array}{l}85,4 \\
85,1 \\
52,6\end{array}$ & $\begin{array}{l}84,6 \\
82,8 \\
56,5\end{array}$ & $\begin{array}{l}84,6 \\
83,4 \\
49,0\end{array}$ & $\begin{array}{l}83,8 \\
82,0 \\
56,8\end{array}$ & $\begin{array}{l}85,0 \\
83,8 \\
56,4\end{array}$ \\
\hline
\end{tabular}

(1) Valeurs non différentes par égalisation expérimentale des quantités consommées.

(2) g de gain de poids/g de protéines consommées.

Les valeurs moyennes des coefficients d'utilisation digestive apparente ont été calculées pour l'ensemble des 3 périodes et sur 6 animaux par lot.

Les valeurs des coefficients pour la matière sèche et pour l'azote sont élevées pour tous les régimes qui sont en général bien utilisés. Cependant aucune des valeurs trouvées ne diffère significativement; on peut seulement noter une tendance, pour le régime $\mathrm{E}_{140}$, du maîs échauffé à entraîner une diminution de la digestibilité de la matière sèche et des protéines de la ration.

\section{2. - Expérience sur rats}

a) Performances pondérales.

Les gains de poids moyens sont rapportés au tableau 5 au cours de deux périodes, 1a première très courte ( 3 jours après le début de distribution), puis sur l'ensemble de la période expérimentale.

Le gain de poids est significativement plus faible pour les lots à base de maîs 
TABLEAU 5

Performances pondérales moyennes des rats soumis aux différents régimes

\begin{tabular}{|c|c|c|c|c|c|c|}
\hline \multirow{2}{*}{ Régime } & \multicolumn{3}{|c|}{$80^{\circ}$} & \multicolumn{3}{|c|}{$140^{\circ}$} \\
\hline & $\mathrm{R}$ & $\mathrm{E}$ & $\mathrm{F}$ & $\mathrm{R}$ & $\mathrm{E}$ & $\mathrm{F}$ \\
\hline Gain total (g) $0-3$ jours & $6,1^{c}$ & $4,8^{a b}$ & $6.7^{b c}$ & $8.7^{c}$ & $3,2^{a}$ & $5,8^{b}$ \\
\hline Gain total (g) $0-3$ semaines & $86,8^{c}$ & $72,3^{b *}$ & $82,8^{c}$ & 91,2 & $55,8^{a *}$ & $79,8^{b c}$ \\
\hline Consommation d'aliment & & & & & & \\
\hline$g(0-3$ jours $) \ldots \ldots \ldots$ & $28,1^{c}$ & $22,0^{a}$ & $74,7 a b$ & $28,4^{b}$ & $22,1^{a}$ & $23,1^{a}$ \\
\hline $\begin{array}{c}\text { Indice de consommation } \\
0-3 \text { semaines } \ldots \ldots \ldots\end{array}$ & $3,30^{a b}$ & $3,51^{b}$ & $3,14^{a}$ & $3,22^{a}$ & $4,21^{c *}$ & $3,32^{a b}$ \\
\hline $\begin{array}{c}\text { Coefficient d'efficacité protidique } \\
0-3 \text { semaines } \ldots \ldots \ldots \ldots\end{array}$ & $3,13^{b}$ & $2,68^{b}$ & $2,96^{c d}$ & $2,86^{c}$ & $2,29^{a}$ & $3,04^{d}$ \\
\hline
\end{tabular}

* Interaction température $\times$ mode de conservation significative.

échauffé $\left(\mathrm{E}^{\mathrm{80}}, \mathrm{E}^{140}\right)$, dès le début de l'expérience ; les résultats sont ensuite confirmés. Par contre, l'effet de la température est à peu près nul, de même que l'on n'observe pas de différences entre les lots $\mathrm{R}$ et $\mathrm{F}$.

Il existe cependant une interaction significative entre le mode de séchage et la température : le maïs échauffé entraîne des performances d'autant plus médiocres qu'il est ensuite soumis à une température de séchage plus forte.

Les quantités d'aliments consommées montrent des variations semblables suivant les lots et les effets apparaissent également dès le début de l'expérience.

C'est enfin le maïs échauffé quii entraîne les plus mauvais indices de consommation, en même temps que la plus faible croissance et la plus faible quantité d'aliment consommée. On constate de plus dans les mêmes lots une diminution du coefficient d'efficacité protidique.

b) Utilisation digestive et métabolique des rations.

Les valeurs du coefficient d'utilisation digestive de la matière sèche et de la matière organique varient peu en fonction du régime (tabl. 6).

Par contre on observe un effet significatif dépressif de la température sur la digestibilité des protéines. Ainsi, les rations à base de maïs chauffé à $I 40^{\circ} \mathrm{C}$ présentent une digestibilité moyenne des protéines d'environ $4 \mathrm{p}$. Ioo inférieure à celles contenant le maïs chauffé à $80^{\circ} \mathrm{C}$.

Il faut, d'autre part, souligner que l'azote de la ration est utilisée de façon différente suivant la température de chauffage, mais aussi suivant le procédé de conservation. Le coefficient d'utilisation pratique de l'azote (tabl. 6) traduit globalement cette tendance dont la signification statistique n'a pu être calculée en raison de l'analyse globale par lot des carcasses de rats. 
TABLEAU 6

Utilisation digestive des principaux éléments de la vation pour le Rat. Calcul du coefficient d'utilisation pratique de l'azote

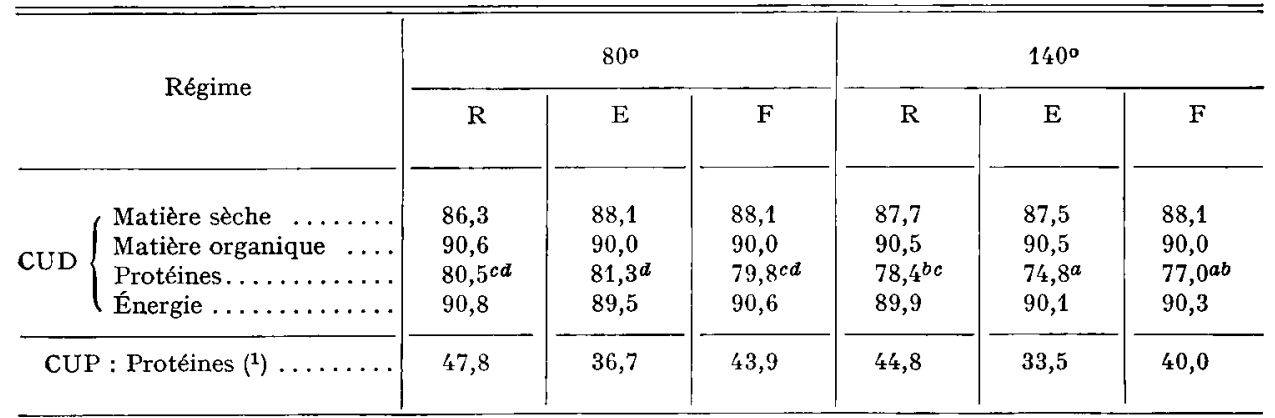

(1) Déterminé globalement sur chaque lot de 9 rats; aucun calcul statistique n'est alors possible.

\section{IV. - DISCUSSION ET CONCLUSION}

\section{I. - Influence des procédés de conservation sur les performances zootechniques}

D'une façon générale, la température de séchage influe peu ou pas sur la vitesse de croissance du porcelet ou du rat, quelle que soit la période considérée. Les résultats sur porcelets sont en assez bon accord avec ceux de JENSEN et al. (Ig6o) qui avaient limité les essais à une température voisine de $1^{\circ} 0^{\circ} \mathrm{C}$ pour le séchage. Par contre, pour le Rat, ils sont en opposition avec ceux de HATHAWAY et al. (I952), relatant un effet significatif de la température de séchage, mais pour une vitesse de croissance des animaux beaucoup plus faible que celle que nous observons. On constate une différence de réponse entre le porcelet et le rat, qui peut s'expliquer en raison de la composition différente des régimes utilisés : le rat recevait une ration à 93,5 p. Ioo de mais, alors que le porcelet recevait seulement 67,5 p. Ioo. La méthodologie utilisée avec le rat permet d'augmenter la précision du test de la valeur du maïs pour assurer une bonne croissance chez le jeune animal.

Un effet important de l'échauffement avant séchage a été observé d'autre part ; la dépression de la vitesse de croissance varie de 5 à Io p. Ioo sur le porcelet et de I7 à $39 \mathrm{p}$. Ioo chez le rat (tab1. 7) par rapport au maïs témoin séché dès la récolte. L'altération due à l'échauffement est d'autant plus forte que la température de séchage est ensuite élevée.

Parallèlement un effet défavorable de certains traitements est observé sur la quantité d'aliment consommée (tabl. 7). La diminution de la vitesse de croissance pour certains lots peut être expliquée en partie par la diminution de la consommation de l'aliment, observée à la fois chez le porcelet et le rat. Ces résultats sont en accord avec ceux de HATHAwAy et al. (I952) sur le rat et avec ceux de JENSEN et al. (I960) chez le porc, qui avaient démontré une diminution de l'appétibilité des rations à

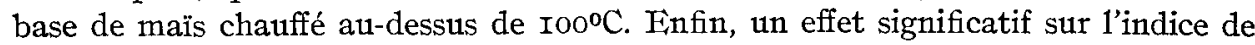


consommation de l'aliment (tabl. 7) s'ajoute au précédent. Ces deux effets constatés permettent d'expliquer la quasi totalité de la variation des performances des deux catégories d'animaux.

\section{TABLEAU 7}

Effet de l'échauffement du maïs sur les performances relatives des animaux (croissance et consommation d'aliment)

\begin{tabular}{|c|c|c|c|c|c|}
\hline \multirow[b]{2}{*}{$\begin{array}{l}\text { Différences entre } \\
\text { régimes }\end{array}$} & \multicolumn{2}{|c|}{ Période $0-3$ jours } & \multicolumn{3}{|c|}{ Période totale } \\
\hline & $\begin{array}{l}\text { Gain de } \\
\text { poids } \\
(\%)\end{array}$ & $\begin{array}{l}\text { Consom- } \\
\text { mation } \\
(\%)\end{array}$ & $\begin{array}{l}\text { Gain de } \\
\text { poids } \\
(\%)\end{array}$ & $\begin{array}{l}\text { Consom. } \\
\text { mation } \\
(\%)\end{array}$ & $\begin{array}{l}\text { I. C. } \\
(\%)\end{array}$ \\
\hline $\begin{array}{l}\text { Porcelet : } \\
\mathrm{R}_{\mathbf{8 0}} \text { et } E_{80} \ldots \ldots \\
\mathrm{R}_{140} \text { et } E_{140} \ldots\end{array}$ & & & $\begin{array}{l}9,7 \\
5,0\end{array}$ & $\begin{array}{l}4 \\
8\end{array}$ & $\begin{array}{l}7,4 \\
8\end{array}$ \\
\hline $\begin{array}{l}\text { Rat : } \\
R_{80} \text { et } E_{80} \ldots \\
R_{140} \text { et } E_{140}\end{array}$ & $\begin{array}{l}43 \\
64\end{array}$ & $\begin{array}{l}22 \\
23\end{array}$ & $\begin{array}{l}17 \\
39\end{array}$ & $\begin{array}{l}12 \\
21\end{array}$ & $\begin{array}{l}11 \\
13\end{array}$ \\
\hline
\end{tabular}

\section{2. - Principaux effets sur la digestibilité des éléments de la ration}

La diminution du coefficient d'efficacité protidique des rations à base de maïs échauffé est due vraisemblablement à une diminution de la disponibilité des protéines de la ration pour les deux types d'animaux, ainsi que l'avaient supposé CABEL, et al. (I958) et HATHAWAy et al. (I952) dans le cas d'un chauffage excessif.

Cependant, les résultats concernant les coefficients de digestibilité observés sur le porcelet ne permettent pas de conclure à un effet dépressif de la température ou même de l'échauffement du maïs sur l'utilisation digestive des éléments de la ration. Ceci est probablement dû à la valeur biologique élevée du complément protidique utilisée par le porcelet car le maïs apporte seulement $20 \mathrm{p}$. Ioo des protéines totales de la ration. Chez le Rat où le maïs apporte $80 \mathrm{p}$. Ioo des protéines totales, on observe des différences marquées sur la digestibilité des protéines. Par contre, 1a digestibilité de 1'énergie est peu affectée par la température et les divers traitements.

L'effet néfaste d'une température élevée sur la disponibilité des protéines de certains produits pour l'animal est bien connu (CARPENTER et al., Ig62 ; RÉRAT et LougNoN, I963; HENK et LAUBE, I959). Il en résulte que l'utilisation globale des protéines du mais pour la synthèse protidique est diminuée, comme l'indiquent globalement les valeurs du coefficient d'utilisation pratique de l'azote, lorsque la température de séchage est trop forte, ou que le maïs a subi un échauffement.

Enfin, la diminution du coefficient de digestibilité de l'énergie du régime, constatée chez le rat, peut s'expliquer par la carbonisation partielle de certains grains de maïs séchés à $140^{\circ} \mathrm{C}$. 


\section{3. - Conclusion}

La température de séchage, dont l'effet n'apparaît pas sur le porcelet, est décelée chez le Rat recevant une grande quantité de maïs dans sa ration. La diminution de digestibilité des protéines provoquée par une trop forte température nous indique les précautions à prendre lors de la pratique du séchage des grains et les conséquences éventuelles d'une surchauffe accidentelle. L'échauffement des grains en raison de son effet dépressif sur les performances zootechniques des animaux paraît être à proscrire.

Sur le plan méthodologique et à condition de rééquilibrer la ration en certains acides aminés essentiels, le rat paraît être un animal de choix pour le test de la valeur alimentaire du maïs grain séché pour le Porc. La sensibilité du test paraît excellente, en bonne corrélation avec les résultats obtenus sur porcelets. D'autre part, la rapidité du test paraît intéressante à souligner : les résultats définitifs peuvent être acquis 3 jours après la distribution du régime.

\section{SUMMARY}

\section{VIII. - NUTRITIONAL VALUE AND DIGESTIBILITY OF MAIZE-GRAIN FOR PIGLETS AND RATS}

The study of the nutritional value of the 6 samples of maize has been carried out on weanling piglets and rats.

The influence of the storage conditions and temperature processing was determined by measuring the mean performances of the animals (daily weight gain, food consumption, food conversion and scouring of piglets). The mean apparent digestibility was also measured for the major nutrients of the diet. The processing temperature has a slight or no effect on the mean performances and digestibility of all nutrients for piglets. On the other hand, a significant depressive effect on the protein digestibility is found in rats when they receive a high maize concentrated diet (93,5 p. I0O) after the $140^{\circ} \mathrm{C}$ drying processes.

The most important effect is observed with the spontaneous heated maize diet since it reduces the mean weight gain of piglets and rats from 5 to $40 \mathrm{p}$. IOO, in comparison with the control group. These results can be explained both by a decrease in the mean food intake $(4$ to $20 \mathrm{p}$. roo less) and an increase in the food conversion ratio (to $33 \mathrm{p}$. 100, according to table 7 ).

Conclusions are drawn with regard to grain processing after harvesting and the animal's sensitivity to the alteration of maize during different kinds of storage. The laboratory animals (Rat) are very quickly able to appreciate after 3 days of experiment, the grain damage and altered nutritional value.

\section{RÉFÉRENCES BIBLIOGRAPHIQUES}

Cabell C. A., Davis R. F., Saul R. A., 1958. Some effects of variation in drying temperature, heating time, air flow rate, and moisture content on nutritive value of field shelled corn. J.Anim. Sci., 17, 1244.

Carpenter K. J., Morgan C. B., Lea C. H., Parr L. J., I962. Chemical and nutritional changes in stored herring meal. 3-Effect of heating at controlled moisture contents on the binding of amino acids in freeze-dried herring press cake and in related model systems. Brit. J. Nutr., 16, 45 I-465.

Cochran W. G., Cox G. M., r962. Experimental designs. John Wiley and sons, 6ri p., New York.

Hathaway I. L., YUnG F. D., Kiesselach T. A., I952. The effect of drying temperature upon the nutritive value and commercial grade of corn. J. Anim. Sci., 11, 430-440. 
HENK G., LAUBE W., 1959. Untersuchungen zur Heisslufttrocknung. 3-Mittleitung Ursachen und Kennzeichnung der Verdaulichkeitsminderung. Archiv. Tiernähr., 19, 289-298.

HENRY Y., 1968. (Communication personnelle).

JENSEN A. H., TERRIL S. W., Becker D. E., I960. Nutritive value of corn dried at $140^{\circ}-180^{\circ}$ and $220^{\circ}$ Fahrenheit for swine of different ages. J. Anim. Sci., 19, 629-638.

Rerat A., Lougnon J., r963. Influence de la conduite du séchage à la flamme sur l'efficacité des protéines de farine de hareng. Études sur le Rat. Ann. Biol. anim. Bioch. Biophys., 3, h. s., 7r-74. 